\title{
Clip and snare countertraction technique for rectal submucosal dissection
}

\section{Georgios Mavrogenis ${ }^{a}$, Nikolaos Georgousis ${ }^{a}$, Dimitrios Ntourakis ${ }^{b}$, Anastasios Mavrogiorgisc}

Gastroenterology and Endoscopy Center of Mytilene, Mytilene, Lesvos; European University of Cyprus, Nicosia, Cyprus; Vostaneio Hospital, Mytilene, Lesvos, Greece

A 62-year-old woman was scheduled for screening colonoscopy. Endoscopy revealed a flat erythematous zone of $2 \times 2 \mathrm{~cm}$ of the distal rectum with a $2-\mathrm{mm}$ expansion into the anal canal (Fig. 1). Biopsies were consistent with an adenomatous lesion with low-grade dysplasia. In view of the location of the lesion, we decided to resect it by means of endoscopic submucosal dissection. The lesion was lifted with a mixture of $500 \mathrm{~mL}$ of $6 \%$ hydroxyethyl starch 130/0.4 in $0.9 \%$ sodium chloride injection (Voluven ${ }^{\oplus}$, Fresenius Kabi Hellas A.E.), $2 \mathrm{~mL}$ of indigo carmine, $1 \mathrm{mg}$ of adrenaline, and $75 \mathrm{mg}$ of ropivacaine. After a circumferential incision and trimming of the periphery, a clip (EZ Clip, Olympus) was placed on the mucosal flap of the anal side of the lesion. A polypectomy snare was then looped over the tip of the endoscope and released over the deployed clip. The snare secured the proximal part of the clip and the handle was fixed in the closed position. Thus, pushing or pulling the catheter of the snare applied controllable back and forward countertraction. At the end of the procedure, the snare was retracted to retrieve the resected specimen. Endoscopists should be aware of this low-cost and readily accessible countertraction technique that

${ }^{\mathrm{a}}$ Gastroenterology and Endoscopy Center of Mytilene, Mytilene, Lesvos, Greece (Georgios Mavrogenis, Nikolaos Georgousis); Department of ${ }^{\mathrm{b}}$ Surgery, European University of Cyprus, Nicosia, Cyprus (Dimitrios Ntourakis); 'Histopathology, Vostaneio Hospital, Mytilene, Lesvos, Greece (Anastasios Mavrogiorgis)

\section{Conflict of Interest: None}

Correspondence to: Georgios Mavrogenis, Kavetsou 10, 81132 Mytilene, Lesvos, Greece, Tel.: +30 22510 55557, Fax: +30 22510 55557, e-mail: mavrogenis@gmail.com

Received 17 July 2016; accepted 26 July 2016

Published online 6 September 2016

DOI: http://dx.doi.org/10.20524/aog.2016.0081

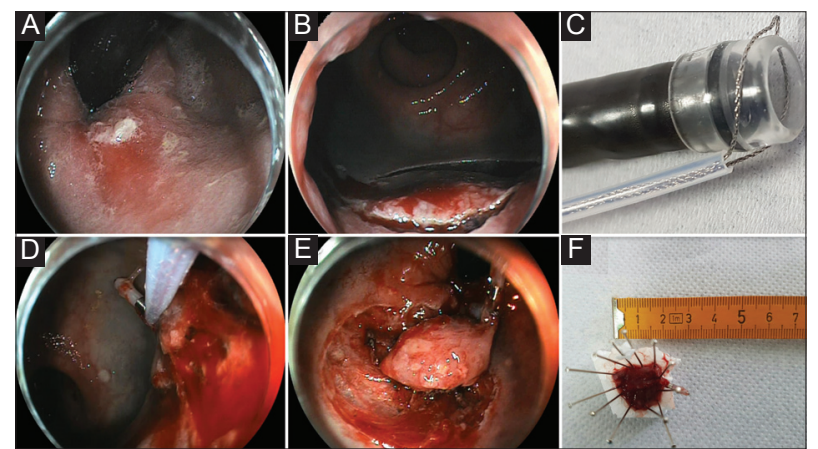

Figure 1 (A) Erythematous flat lesion of the lower rectum examined after chromoendoscopy with acetic acid. (B) Circumferential incision with DualKnife (Olympus). (C, D) A snare is looped over the hood of the endoscope and then placed and tightened over a clip at the anal side of the lesion. (E) Pulling or pushing the snare changed the axis of countertraction as desired. (F) Final photo of the resected specimen.

allows bidirectional traction, the possibility of snare removal when no further countertraction is needed, and finally retrieval of the resected specimen. Although initially described in a case series of gastric endoscopic submucosal dissection, the clip and snare technique proved its efficacy in shortening the overall duration of the procedure in a recent comparative study from Japan including 17 colorectal lesions [1-2].

\section{References}

1. Baldaque-Silva F, Vilas-Boas F, Velosa M, Macedo G. Endoscopic submucosal dissection of gastric lesions using the "yo-yo technique". Endoscopy 2013;45:218-221.

2. Yamada S, Doyama H, Ota et al. Impact of the clip and snare method using the prelooping technique for colorectal endoscopic submucosal dissection. Endoscopy 2016;48:281-285. 